\title{
Laryngotracheoesophageal cleft type 0
}

INSERM

\section{Source}

INSERM. (1999). Orphanet: an online rare disease and orphan drug data base.

Laryngotracheoesophageal cleft type 0. ORPHA:280205

Laryngo-tracheo-esophageal cleft (LC) type 0 is a congenital respiratory tract anomaly characterized by a submucosal laryngo-tracheo-esophageal cleft with minor symptoms or an asymptomatic course. 